\title{
The nuclear microprobe used to investigate meteorites
}

\author{
R.D. Vis*, A.C. Kik, J.L.A.M. Kramer \\ Faculty of Physics and Astronomy, Vrije Universiteit, De Boelelaan 1105, 1081 HV Amsterdam, The Netherlands
}

\begin{abstract}
During many years a growing group of researchers has been involved in the study of meteorites. Meteorites are the only objects originating from the solar system in its early stage and accessible to detailed research. Research on meteorites has been done with a wide variety of analytical techniques for the investigation of their chemical composition, structure and texture, presence of isotopic anomalies, radioactivity and so on. Since the development of the nuclear microprobe, this instrument also has been and is used as a probe for the investigation of meteorites. In this contribution, results of the ongoing research with the nuclear microprobe on meteorites is described.
\end{abstract}

\section{Introduction}

A substantial part of the information on the early solar system comes from the investigation of meteorites. The chemical composition has been extensively studied with bulk analytical techniques to determine major-, minorand trace-elements. The lateral distribution of majorand minor-elements are measured with the electron microprobe, while Secondary Ion Mass Spectrometry (SIMS) has been used to determine isotopic ratios of several elements. The advent of the nuclear microprobe with its possibilities of ion-beam analysis on a $\mu \mathrm{m}$-scale adds a valuable tool to further and more detailed analysis of extra-terrestric samples. These studies can provide a more detailed knowledge of processes that took place during the formation of our solar system.

From the main types of meteorites, chondrites are the most interesting as they are considered to be the most primitive material. Especially carbonaceous chondrites, named after their relatively high C-concentration, are one of the oldest objects available for research. Both these chondrites and the so-called ordinary chondrites are characterized by the presence of chondrules, millimeter sized spherical objects, which often make up more than $50 \%$ of the chondritic material.

The ordinary chondrites are further subdivided into subgroups, according to their bulk chemical composition, more especially the $\mathrm{Fe} / \mathrm{Si}$ ratio, and according to the

\footnotetext{
* Corresponding author.
}

amount of alteration that took place after the formation of the objects. The latter subdivision is into petrologic types. For a more detailed description of the classification see for instance Ref. [1].

Meteorites have been studied with a very wide variety of methods. It is outside the realm of this paper to review all methods and chemical data available. We restrict ourselves to data obtained with a nuclear microprobe. Apart from early work at Los Alamos and early and recent work at Heidelberg (see below) no nuclear microprobes have been used for systematic studies on meteorites so far. Therefore, the experimental sections describe mainly work performed at Amsterdam. The experimental procedures, however, are strongly related to procedures used to study terrestrial geological samples as done at many places and are comparable with analytical methods used elsewhere.

\section{Possible contributions from the nuclear microprobe}

\subsection{Chemical information}

The most obvious contribution one can expect from the nuclear microprobe is information about the distribution of elements present in low concentration. The distribution of major elements in order to establish the mineralogy is the territory of the electron probe; trace level elements are accessible with the nuclear microprobe using micro-PIXE. Mainly the, in geochemical respect, volatile elements are studied. These studies can give an understanding about the temperatures and perhaps also 
condensation rates during formation. A particularly interesting, element in this respect is Carbon, because the C-chemistry is very sensitive to parameters such as temperature and pressure. Carbon distributions have been measured with deuteron-beams, using the ${ }^{12} \mathrm{C}(\mathrm{d}, \mathrm{p}){ }^{13} \mathrm{C}$ nuclear reaction.

Volatile elements can also indicate the difference in temperature experienced by the matrix of the chondrites and the chondrules. If chondrules really are formed by a high temperature event of, yet, unknown origin, then a depletion of these volatiles is to be expected.

Measurements of a large number of matrices from ordinary chondrites from a low petrologic (least altered) type may support the assumption that these meteorites have a common matrix as suggested by Anders [2]. To avoid the cumbersome separation between matrix and chondrules, the nuclear micro-probe is a very helpful instrument to select particular fine-grained matrix areas. Rare earth elements also are measurable with microPIXE. Jones et al. [3] investigated phosphates in the Shergotty basaltic meteorite, in order to find out if, as suggested by Jagoist? et al. [4] the clock system that is based on Sm-Nd had been reset by hydrothermal alteration. Also planetary dust particles $[5,6]$ and iron meteorites [7] have been studied using micro-PIXE.

\subsection{Isotopic information}

For a number of elements, it is possible to determine the ratio of the isotopes. Especially in combination with micro-PIXE to investigate the local mineralogy, the in situ determination of these ratios opens up unique possibilities to search for remnants of a super-nova explosion that is thought to be a trigger for the formation of the solar system [8].

Obviously, nuclear reactions have to be used for this purpose, which immediately limits these applications to low $Z$ elements.

Most interesting in this respect are the isotopes of $\mathrm{C}, \mathrm{O}$ and $\mathrm{Mg}$. Very intruiging is the recent finding by Engel et al. [9] of highly enriched concentrations of ${ }^{13} \mathrm{C}\left(\delta^{13} \mathrm{C}=+3 \%\right)$ in individual am $\mathrm{As}$ in Murchison, a carbonaceous chond , i , . there is different enrichment $b$ cin the optical stereoisomers of alanine, which may ead to speculations that pre-biotic material with its preference for $\mathrm{L}$-amino-acids has been made already during the super-nova shortly before the formation of our solar system. The asymmetry might be induced by intense polarised radiation such as synchrotron radiation.

Oxygen-isotopic ratios are also interesting. An excess of ${ }^{16} \mathrm{O}$ is also indicative for supernova condensates. The very heterogeneous distribution of the isotopic anomalies of oxygen has been explained by later diffusion and partial exchange with the environment.
An excess of ${ }^{26} \mathrm{Mg}$ over the other two stable isotopes is informative, because it can point towards the decay of ${ }^{26} \mathrm{Al}$ (half life $0.74 \mathrm{My}$ ) that has been made in large quantities. This $\beta$-decay has been one of the major local heat sources in the early stage of the solar nebula.

\section{Experimental}

Experimental techniques to apply the nuclear microprobe to study stony meteorites are not much different than applications in geology, exploring and analyzing terrestrial samples. Silicate reference standards can be used for system calibration [10]. To calibrate nuclear reactions on Mg-isotopes, forsterite has been used.

\subsection{Sample preparation}

Although targets are necessarily thick with respect to the beam as it is impossible to prepare slices that can be analysed as thin targets, it is very helpful to polish meteoritic samples down to $10-30 \mu \mathrm{m}$. Such sections are light-transparant, except for opaque minerals, provided that they are mounted on a transparant backing, which enables proper optical microscopy in transmitted light and proper positioning of the beam on target. We use Herasil backing, which is a high purity quartz, just to be sure that if the beam hits the backing through cracks or other inhomogeneities or simply through a thin section, no contribution is given to the X-ray spectrum other than Si. All samples are coated with carbon to prevent charging. All samples are photographed in detail for orientation under the microscope. It has been proven to be very helpful to determine the mineralogy with an electron microprobe (EMP), to have elemental maps of the major element at hand before micro-PIXE measurements start. A detailed quantitative comparison between the EMP and the nuclear microprobe is, however, often disappointing, due to the very different penetration depth of both beams.

\subsection{Irradiation}

The NEC 1.7 MV tandem accelerator, its beam lines and the Amsterdam microprobe are described elsewhere [11]. All results described below are obtained with a relatively large beam spot $( \pm 10 \mu \mathrm{m})$ in order to have currents up to $1 \mathrm{nA}$. The energy used for PIXE measurements is $3.4 \mathrm{MeV}$, relatively high but with the advantage of obtaining also information on $\mathrm{Mg}, \mathrm{Al}$ and $\mathrm{Si}$ by their gamma-radiation emitted due to $\left(p, p^{\prime} \gamma\right)$-type nuclear reactions. Although this information can be obtained also by PIXE, we have adopted this method to enable to use thick absorbers with subsequent loss of information of low $Z$ elements, in front of the $X$-ray detector, which 
prevents pile-up and enables measurement of low concentrations of the heavier volatiles. For the determination of ${ }^{18} \mathrm{O}$, a beam of $860 \mathrm{keV}$ protons has been used to benefit from the resonance in the ${ }^{18} \mathrm{O}(\mathrm{p}, \alpha){ }^{15} \mathrm{~N}$ reaction. For the determination of $\mathrm{C}$, a deuteron micro-beam of 1.2-1.4 MeV has been used.

\subsection{Detection and data-acquisition}

During irradiation, $\mathrm{X}$-rays are detected with a $\mathrm{Si}(\mathrm{Li})$ $\mathrm{X}$-ray detector; backscattered protons are detected with a surface barrier detector, placed at the backward angle of $150^{\circ}$ and a high purity Ge-detector for the detection of $\gamma$-rays.

Our microprobe is equipped with a current monitor, consisting of a thin $(100 \mathrm{~nm}) \mathrm{Au}$ foil that is put in the beam upstream of the sample during a well known percentage of the time. A second surface barrier detector views this foil and records accumulated charge after proper calibration.

During the measurements of $\mathrm{C}$ or $\mathrm{O}$, annular surface barrier detectors are placed in front of the specimen in order to provide a large solid angle with a near to $180^{\circ}$ geometry.

The real time data-acquisition system consists of a VMEbus which reads out a CAMAC crate and a SUN workstation for monitoring of spectra, distributions, etc. [12]. Datid storage is in list mode for a maximum of $4 \mathrm{ADC}$ 's and $1 \mu \mathrm{m}$ resolution.

\subsection{Data reduction and analysis}

After irradiation spectra per pixel are constructed for all detectors. X-ray spectra are futher analysed with the GUPIX computcr code [13], which yields clemental concentration maps. For a high number of PIXELS this is a time consuming procedure. If more than 100 Pixels are to be analysed, energy bins are defined in the spectra and plotted as a function of position. Gamma spectra are fitted with SPECFIT for the evaluation of peak areas [14]. For ${ }^{24,25,26} \mathrm{Mg}$, excitation functions, or, more precisely, thick target yields between 2.0 and $3.6 \mathrm{MeV}$ has been measured on a forsterite standard (Fig. 1). For the determination of the isotopic ratios of the Mg-isotopes, and/or the Mg-concentration a computer code has been written that takes into account the matrix composition and the stopping power of the sample and standard.

Oxygen isotopic ratios are only obtained from ${ }^{18} \mathrm{O}$ and ${ }^{16} \mathrm{O}$, due to the very low abundance of the odd isotope. ${ }^{16} \mathrm{O}$ is measured by RBS and the spectra are analysed using RUMP; the $\alpha$-particles emitted by

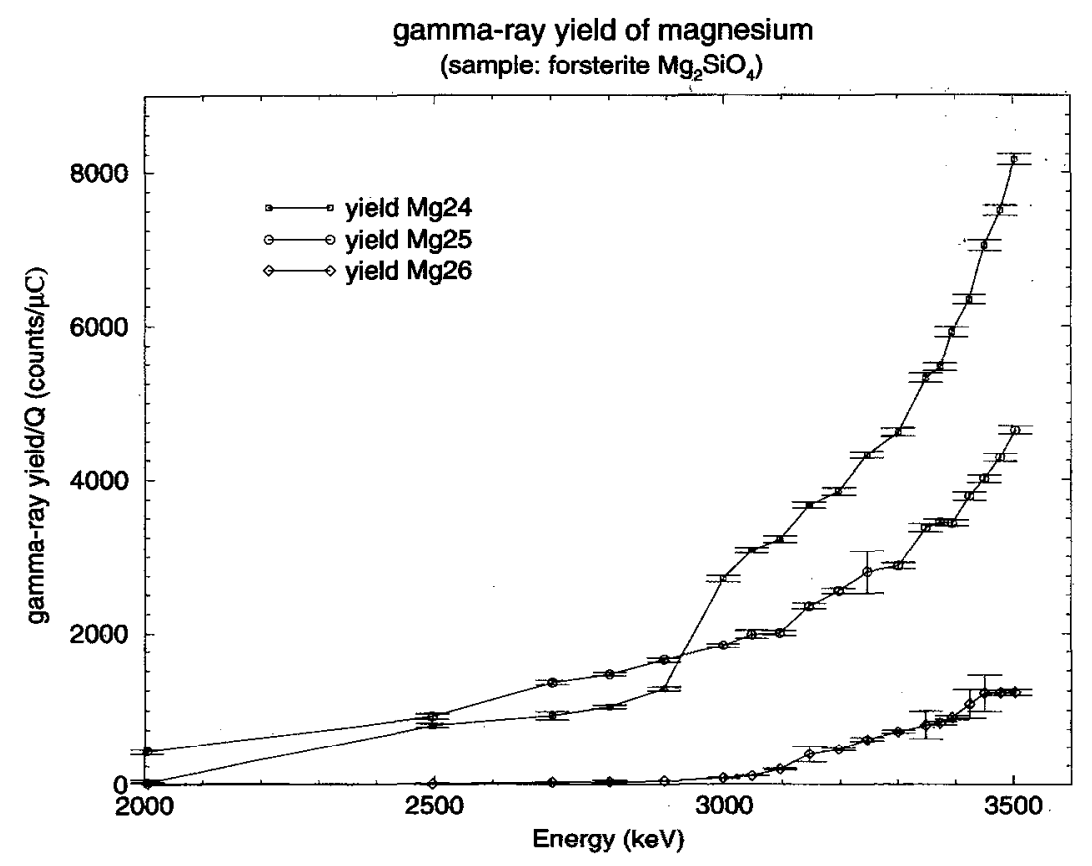

Fig. 1. Thick target yields of the $\left(\mathrm{p}, \mathrm{p}^{\prime} \gamma\right)$ reactions on $\mathrm{Mg}$ isotopes. Used are the 1369,585 and $1809 \mathrm{keV} \gamma$-lines for the detection of respectively ${ }^{24,25,26} \mathrm{Mg}$. 

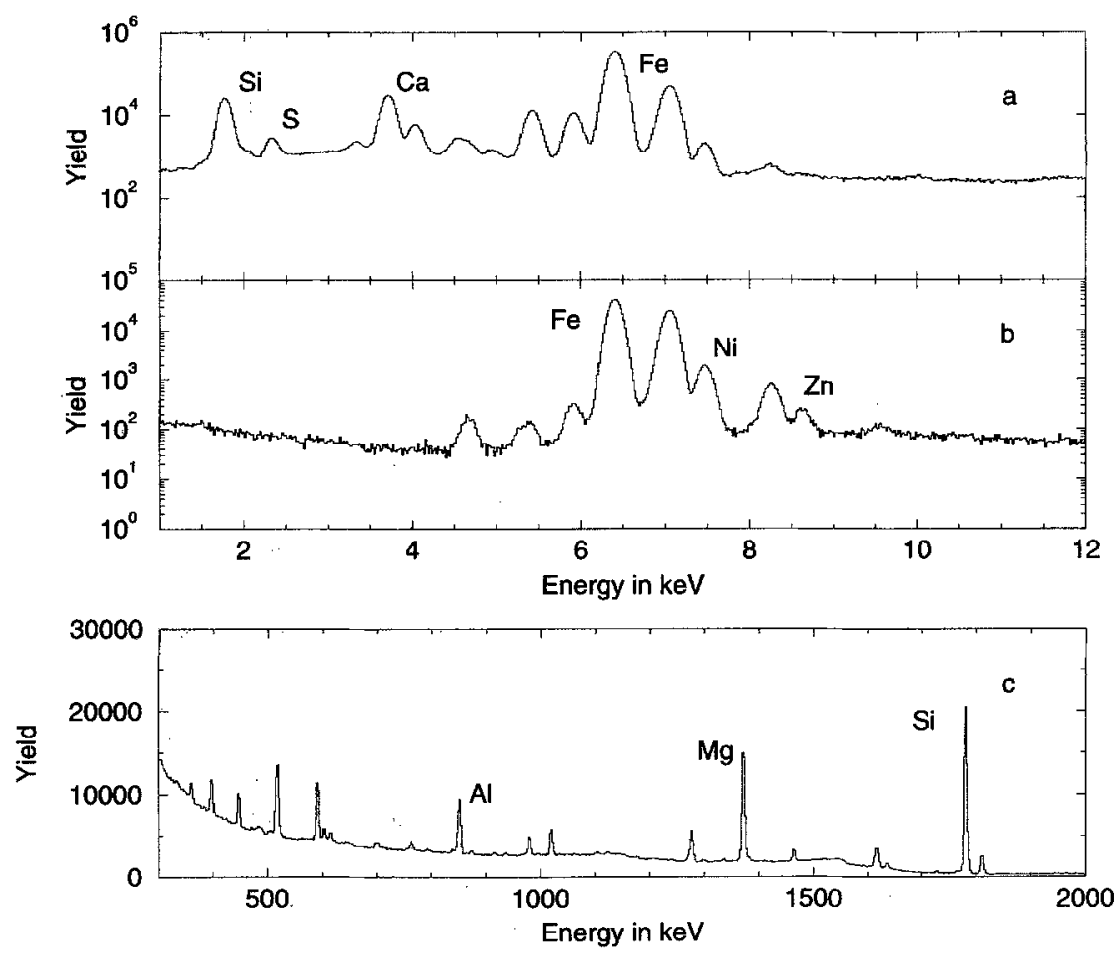

Fig. 2. X-ray and $\gamma$-ray spectra obtained from a chondrule in Inman. Spectrum (a) has been measured without an absorber; (b) with a $170 \mu \mathrm{m} \mathrm{Al}$ absorber. (c) is the $\gamma$-spectrum; $3.4 \mathrm{MeV}$ protons have been used for the excitation; only the most important peaks are marked for orientation.

${ }^{18} \mathrm{O}$ are analysed by just putting a window in the spectrum, as at that energy the spectra are virtually background free. The same holds for the protons emitted during the nuclear reaction ${ }^{12} \mathrm{C}(\mathrm{d}, \mathrm{p}){ }^{13} \mathrm{C}$ $[15,16]$. Concentration assignment in these cases is done in a relative way by comparison with suitable standards.

\section{Results}

\subsection{Volatile elements}

Bajt et al. [17] measured trace element profiles in crystals inside chondrules and concluded that this trace element zoning varics strongly, even within the same chondrule. The authors measured concentration distributions of a number of elements in single crystals of olivine in chondrules from both Semarkona (LL3.0) and Dhajala (H3.8). Micro-PIXE was used and the measurements were performed with the Heidelberg nuclear microprobe. From their data they concluded that the abundance of volatile elements and the observed crystal structures and textures suggest that chondules experi- enced high temperatures only for a very short time. Experiments in which chondrules were heated to $1800 \mathrm{~K}$ gave heating times of roughly a few minutes [18]. The heating source that caused chondrule formation is unknown and also the question of location of the formation, such as in a very early stage in the gas/dust cloud or as a sort of volcanism in the planetisimals much later, is not completely settled yet.

From carbon profile determinations, performed at Amsterdam, in a large number of ordinary chondrites, Makjanic et al. [19] concluded first of all that all Cconcentrations fall within a relatively narrow bandwidth $(0.03-0.2 \%$ ), supporting the idea of a common matrix material. They also conciuded that the matrix experiences a much lower temperature. This was deduced from the finding that $\mathbf{C}$ was mainly located as a jacket around $\mathrm{Fe}$ or Ni-grains and thus possibly formed by a Fischer-Tropsch chemical reaction reducing oxides to hydrates with the metal as catalyst. This reaction only proceeds at a temperature between 600 and $750 \mathrm{~K}$, substantially lower than the chondrules. The hydrates subsequently form $\mathrm{C}$ by pyrolysis. Although this theory is rather old already, it has recently been questioned by Cronin et al. [20], who measured aliphatic hydrocarbons 

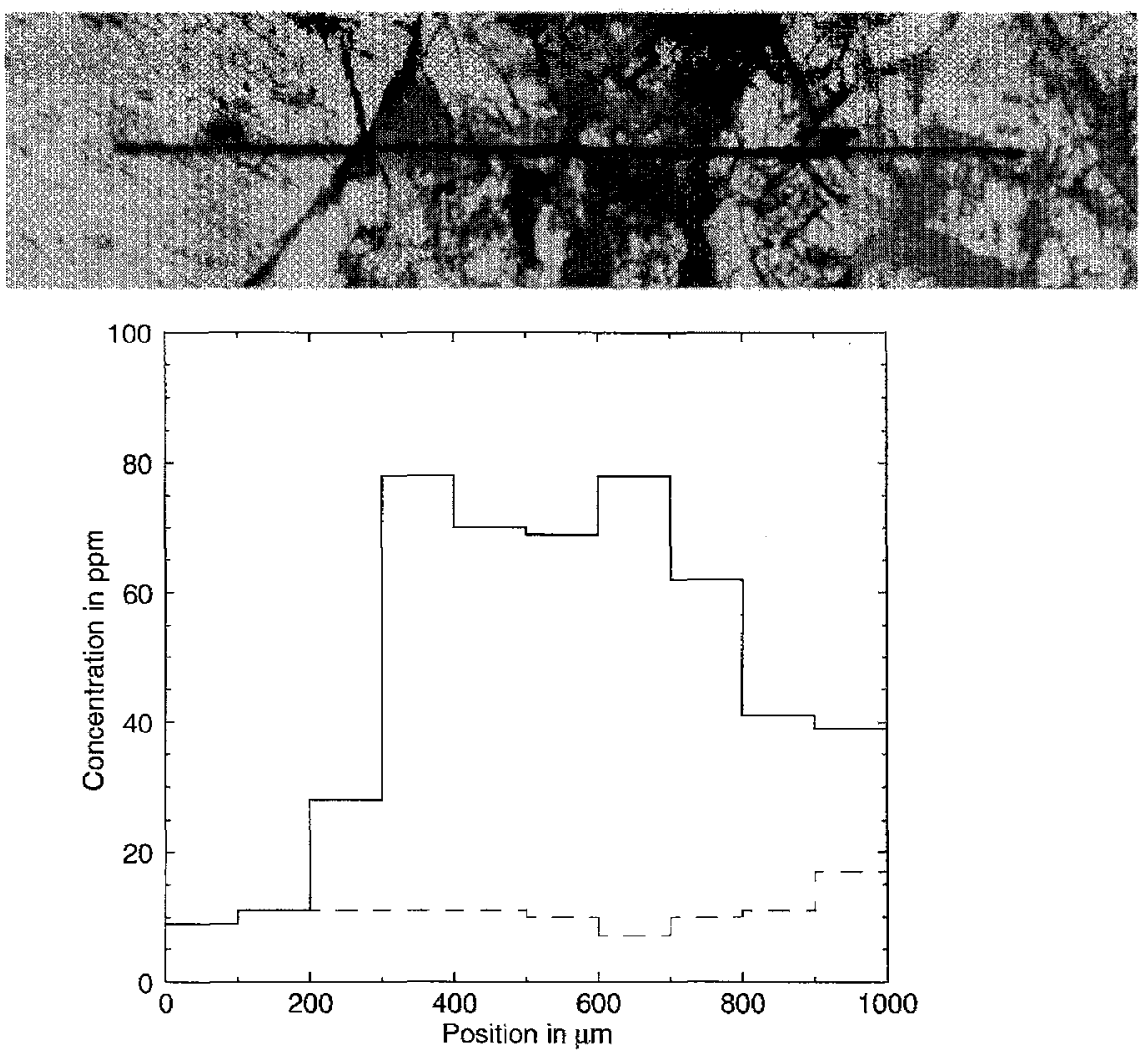

Fig. 3. Results of a line scan made over two chondrules and a matrix area in between. The top photograph shows the scan, which becomes visible by deposition of carbon; the bottom figure shows the distribution of zinc. The dashed line represents the detection limits at the various positions.

in Murchison. Makjanic's data, however, seem convincing and nicely illustrate the possibilities of the nuclear microprobe applying combined micro-PIXE and a nuclear reaction [21].

Zinc has been determined with our nuclear microprobe because $\mathrm{Zn}$ is a good representative of the volatile elements. Especially the question of depletion in chondrules has been addressed.

Line scans have been made over chondrules of Inman (L3) and Chainpur (LL3.4). Fig. 2 shows typical spectra from X-rays (a and b) recorded without (a) and with a $170 \mu \mathrm{m}$ thick Al-absorber and $\gamma$-rays (c). From this combination it has been demonstrated that it is possible to determine the detailed mineralogy along the track by calculating the number of individual atoms of each element on the basis of $6 \mathrm{O}$-atoms for pyroxenes and $4 \mathrm{O}$-atoms for olivines. This enables the deduction of the ratio of individual minerals, the socalled end members, such as enstatite, ferrosilite, etc. $\mathrm{Mg}$ is deduced from the gamma radiation; other elements from PIXE.
In Fig. 3 results from a $1 \mathrm{~mm}$ scan over two chondrules in Inman with matrix in between is presented. It is immediately clear that both chondrules are depleted in $\mathrm{Zn}$, and also that the depletion is different for the two chondrules, which are of a different type, namely porphyritic and radial pyroxene respectively. Unfortunately, the statistics are not sufficient to demonstrate convincingly enrichment at the rims, which would indicate formation of chondrules in situ; more detailed measurements are needed but it is a tedious job to acquire such data, caused both by the low concentration of $\mathrm{Zn}$ and by the spectral interference of the Ni- $\mathrm{K}_{\beta}$ and the $\mathrm{Zn}-\mathrm{K}_{\alpha}$.

In one of the Chainpur chondrules, spots with exceptionally high $\mathrm{Pb}$-concentration were found $[22,23]$. The origin of these spots is not clear yet but upper limits of the concentrations of $U$ and $T h$, in combination with the determination of the ratio of the isotopes ${ }^{204,206,207} \mathrm{~Pb}$ by SIMS, showed that this $\mathrm{Pb}$ has been recently separated from its source. Very likely, this $\mathrm{Pb}$ has been imported in the chondrule by a major collision which broke up the parent body. 


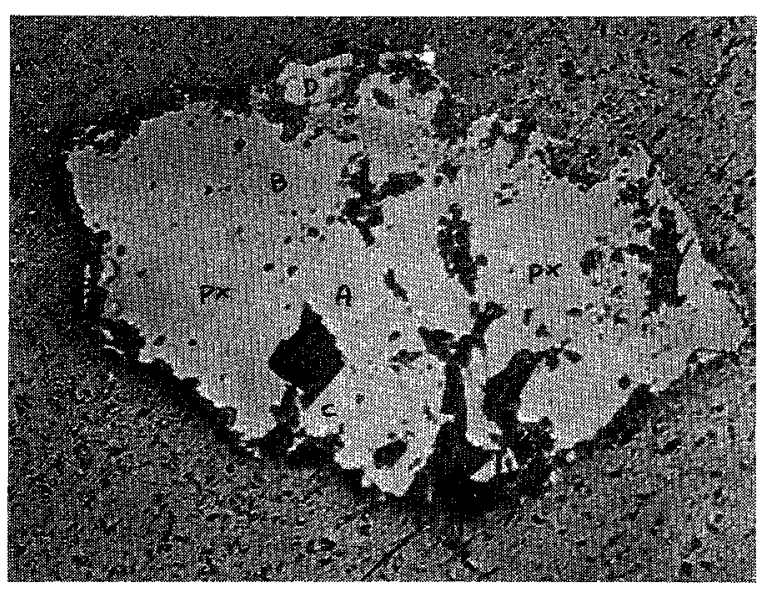

Fig. 4. A photograph of the $\mathrm{Ca}-\mathrm{Al}-$ rich inclusion in Allende, used for O-isotopes measurements. At the marked positions A, $\mathrm{B}, \mathrm{C}$ and $\mathrm{D}$ spot measurements with $860 \mathrm{keV}$ protons has been performed to detect ${ }^{18} \mathrm{O}$ and ${ }^{16} \mathrm{O}$; px indicates areas with pyroxene. The inclusion has been embedded in araldite.

\subsection{Isotopic ratios}

Although much information is available on $\mathbf{C}$ isotopic anomalies in meteorites, no nuclear microprobe work has been described to measure the ${ }^{13} \mathrm{C} /{ }^{12} \mathrm{C}$ ratio in chondrites. This is presumably due to the rather low C-concentration in chondrites. On the other hand, the ratio is an interesting indicator for material that survived the formation of the solar system. In SiC grains enrichment of ${ }^{13} \mathrm{C}$ of a factor 2 is reported, which illustrates that this material withdraws itself from the mixing in the solar nebula. Using the nuclear microprobe, most promising is the $(\mathrm{d}, \mathrm{p})$ reaction at $1.3 \mathrm{MeV}$, which has an acceptable cross section on both isotopes [24]. $\left({ }^{3} \mathrm{He}, \mathrm{p}\right)$ reactions with a beam energy of $2.8 \mathrm{MeV}$ has also been studied [25]. The latter reaction suffers from a considerably lower cross section. Provided that one is able to select with the microprobe the proper grains, it should be feasible to acquire data on this enrichment.

With our microprobe, we measured the ${ }^{18} \mathrm{O} /{ }^{16} \mathrm{O}$ ratio in $\mathrm{Ca}-\mathrm{Al}$ rich inclusions in Allende [26], a carbonaceous chondrite. Point measurements were performed on spinel crystals; ratios were compared with a terrestrial spinel that served as a standard. The average ${ }^{18} \mathrm{O}$ abundance, defined as the ratio of ${ }^{18} \mathrm{O}$ over the total oxygen in the sample, of five measurements taken on the standard yielded $2.041 \%$ as compared with the average on four spinel crystals in Allende of $1.958 \%$, demonstrating the feasibility of the method. Fig. 4 shows the $\mathrm{Ca}-\mathrm{Al}$ rich inclusion with the positions of the measurements; the estimated error in the results is of the order of $2 \%$, which means that the enrichement of ${ }^{16} \mathrm{O}$ in the spinels is significant.
For the measurements of the three stable isotopes of $\mathrm{Mg}$, up to now our conclusion has to bc, that the final precision is of the order of $5 \%$, due to poor statistics in reasonable measuring times and due to errors in the excitation functions and the correction procedures for the stopping of the beam as described by van der Stap et al. [27] and Kik [28]. Work is in progress to improve the situation by using thinner specimens and increasing the solid angle subtended by the $\gamma$-detector. Also the use of an anti-Compton shield around the $\gamma$-detector will improve the experimental conditions.

\section{Conclusions}

With a nuclear microprobe, equipped with a data acquisition system that enables detection of X-rays, $\gamma$ rays and particles simultaneously, many applications in the field of meteoritic research are possible. As a supplemental method to the clcctron microprobe and SIMS, the strong points of this instrument are its trace element capability and the possibilities for the measurement of isotopic ratios using suitable nuclear reactions. For the latter application, a high precision and accuracy is necessary; anomalies are very often small, albeit that on a $\mu \mathrm{m}-$ scale high anomalies can sometimes be found. Studies of this kind can provide data that can lead us towards a better understanding of the very complex phenomena that occurred during the early stages of the formation of our solar system.

\section{References}

[1] H.Y. Mc Sween, Meteorites and their Parent Planets (Cambridge University Press, Cambridge, 1987).

[2] E. Anders, Space Sci. Rev. 3 (1964) 583.

[3] J.H. Jones, T.M. Benjamin, C.J. Duffy, P.S.Z. Rogers, M. Hollander and C.J. Maggiore, Meteoritics 20 (1985) 674.

[4] E. Jagoutz and H. Wänke, XVI Lunar and Planet, Science Conf. Supplement A (1985) 15.

[5] E.K. Jessberger, R. Wallenwein, H. Blank and K. Traxel, Meteoritics 20 (1985) 673.

[6] R.D. Vis, C.C.A.H. van der Stap and D. Heymann, Nucl. Instr. and Meth. B 22 (1987) 380.

[7] S.R. Sutton, J.S. Delaney, J.V. Smith and M. Prinz, Geochimica et Cosmochimica Acta 51 (1987) 2653.

[8] A.G.W. Cameron and J.W. Truran, Icarus 30 (1977) 447.

[9] M.H. Engel, S.A. Macko and J.A. Silfer, Nature 348 (1990) 47.

[10] G.K. Czamanske, T.W. Sisson, J.W. Campbell and W.J. Teesdale, American Mineralogist 78 (1993) 893.

[11] R.D. Vis, J.L.A.M. Kramer, G.H.J. Tros, F. van Langevelde and L. Mars, Nucl. Instr. and Meth. B 77 (1993) 41.

[12] J.L.A.M. Kramer, A.H. Ullings and R.D. Vis, Nucl. Instr. and Meth. B 77 (1993) 62.

[13] J.A. Maxwell, J.L. Campbell and W.J. Teesdale, Nucl. Instr. and Meth. B 43 (1989) 218. 
[14] H.P. Blok, J.C. de Lange and J.W. Schotman, Nucl. Instr. and Meth. 128 (1975) 546.

[15] C.C.A.H. van der Stap, D. Heymann, R.D. Vis and H. Verheul, J. Geophys. Res. 91 (1986) D373.

[16] J. Makjanic, D. Heymann, C.C.A.H. van der Stap, R.D. Vis and H. Verheul, Nucl. Instr. and Meth. B 30 (1988) 466.

[17] S. Bajt and K. Traxel, Nucl. Instr. and Meth. B 54 (1991) 317.

[18] A. Tsuchiyama, H. Nagahara and I. Kushiro, Earth Planet. Sci. Lett. 48 (1980) 155.

[19] J. Makjanic, R.D. Vis, J.W. Hovenier and D. Heymann, Meteoritics 28 (1993) 63.

[20] J.R. Cronin and S. Pizzarello, Geochimica et Cosmochimica Acta 54 (1990) 2859.

[21] J. Makjanic, D. Heymann and R.D. Vis, Nucl. Instr. and Meth. B 54 (1991) 325.
[22] J.L.A.M. Kramer, A.C. Kik and R.D. Vis, XXV Lunar and Planet. Science Conf. Houston, U.S.A. (1994).

[23] J.L.A.M. Kramer, A.C. Kik and R.D. Vis, these Proceedings (ICNMTA'94), Nucl. Instr. and Meth. B 104 (1995) 494.

[24] P.M. Pollard, J.W. Mc Millan and D.J. Malcolme-Lawes, J. Radioanal. Chem. 70 (1982) 349.

[25] D. Bromley and E. Almqvist, Reports Prog. Phys. (1960) 544.

[26] G.P. Meeker, G.J. Wasserburg and J.T. Armstrong, Geochimica et Cosmochimica Acta 47 (1983) 3123.

[27] C.C.A.H. van der Stap, A. Hogenbirk, A.J.J. Bos, R.D. Vis and H. Verheul, Nucl. Instr. and Meth. B 3 (1984) 695.

[28] A.C. Kik, Internal Report, Vrije Universiteit, Amsterdam, 1994. 